\title{
WNT Pathway Activated
}

National Cancer Institute

\section{Source}

National Cancer Institute. WNT Pathway Activated. NCI Thesaurus. Code C129437.

A genetic finding indicating that the WNT signaling pathway is activated. 